\title{
LA MESURE RAPIDE DES BASES GÉODÉSIQUES ;
}

\author{
Par I. (H.-EH, I, ILLALVE I).
}

Exposé sommaire des méthodes. - La détermination précise d'une hase gréodésique utilise des procédés qui, tout en variant beaucoup dans le détail, se ramènent essentiellement à deux types distincts: mais encore ces deux modes d'opérer possèdent-ils un départ commun; la longueur à mesurer étant limitée par deux termes invariablement fixés au sol, et le plus souvent enfoncés au-rlessous de son niveau, on place, dans la verticale du premier terme, l'une des extrimitís d'un étalon aligné dans la direction de la base, et qui en mesure la première portée ; c'est seulement à partir de cette opération que les deux méthodes commencent à diverger.

Dans un système employé surtout à la fin du xvir ${ }^{\mathrm{e}}$ siècle et au début du $\mathbf{x}^{\mathrm{e}} \mathrm{x}^{\mathrm{e}}$, on aligne, à la suite du premier, plusieurs étalons placés à une petite distance l'un de l'autre, puis on détermine leur écartement soit à l'aide d'un coin, soit au moyen d'une languette portée par l'un d'eux, et qu'on fait buter contre l'autre, tandis qu'on lit à la loupe la position de la languette par rapport à la division de l'étalon qui la porte.

L'autre système, qui s'est de plus en plus substitué au premier, utilise un seul étalon, que l'on déplace de sa propre longueur devant un repère marquant successivement son extrémité antérieure et son extrémité postérieure. L'étalon est toujours compris entre deux repères, dont un certain nombre sont alignés le long de la base, ceux devenus inutiles après son passage étant reportés en avant de la section mesurée.

La substitution graduelle de cette méthode à l'ancienne a été entrainée par l'abandon des étalons à bouts et leur remplacement par les étalons à traits. Dans l'emploi des règles à traits fins, le repère transitoire est généralement un microscope micrométrique porté, avec ses organes de réglage, sur un solide trépied de bois.

la hase, servant de départ aux triangles géodésiques, il est avantageux de la choisir aussi longue que possible, ou, plus exacte-

\footnotetext{
"Communication faite à la Socièté françaiso fo Phỵique, séance du 7 juitlet 1 ' 11 .
} 
ment. le lui donner une étendue en proportion avec l'ampleur des operations dont elle fournit l'échelle.

Les bases topographiques ou callastrales dépassent rarement 1 kilumètre, tandis que les bases géodésiques alteignent une moyenne dune dizaine de kilometres.

Iu cours du ve siècle. la mesure de- banes, quelle que lint la méthode générale employée, a subi une double évolution. Dansles trois premiers quartiers du sicele, on a cherché surtout à augmenter la précision des mesures, sans se préoccuper beaucoup du labeur qu'elles imposaient: on est arrivé ainsi à réaliser des opérations tries parfaites, mais extrèmement coûtenses, et dont on était conduit à réduire le nombre autant que possible. $\Lambda$ insi, pour l'ensemble considérable de la géodésie française par exemple, on s'est limité à la mesure de trois bases, situées respectivement près de Paris, de Dunkerque et de Perpignan. Tous les autres points géodésiques ont été atteints par des triangles.

Dans les dernières décades du siècle, on a tenté, au contraire, de simplifier les méthodes de mesure, quitte à sacrifier au besoin un peu de l'exactitude réalisée par les méthodes anciennes; on a pu ainsi répartir son effort sur la détermination d un plus grand nombre de bases, contrólant mieux la triangulation.

Mais un fait domine toutes les fluctuations des idées sur la mesure des basses géodésiques: c'est la préoccupation constante d'évaluer' avec exactitude la température des étalons sur le terrain; "t la détermination de cette variable, dont dépend la longueur de l'instrument de mesure, a toujours été considérée par les géodésiens comme si difficile et si importante quon pourrait prencue dire que l'histoire des appareils de base se confond pratiquement avec celle des précautions prises pour éviter les erreurs de température.

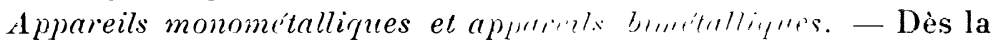
fin du $\mathbf{u}^{\mathrm{e}}$ siècle, deux systèmes distincts detalons se trouvent constamment en présence : les anciens tulom monométalliques accompagnés de thermomètres, et les étalon bimeilalliques, proposés par Borda et Lavoisier, et dam lespuels la longuenr de l'un d'em. considéré comme étalon principal. ul teduite de la diflérence des d.

bans l'emploi de l'étalon monomitallipur. les purtesiens américains ont poussé le perfectionnement at lirextrue en 1 mmergeant la

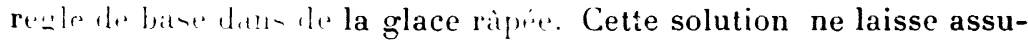


riment rien à désirer au puint de vue théurique; mais elle soulève de wrosses difticult's pratiques en campagne.

Les italın-bimétalliqun- les plus parfaits qui aient été construits sont dus aux frères Brunner, qui ont exécuté-uccersivement les appar.ils de bases d'Espagne. de France, d'Figste et d Allemagne. La

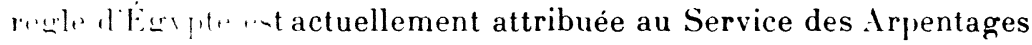
? ce pays; les trois autres ont hervi aux opérations les plus imporinute's de la géodésie européenne.

L'emploi de la règle bimétallique, si l'on veut en tirer tout ce qu'elle peut donner comme précision, exige plus de cinquante hommes sur le terrain, et celte règle permet de mesurer environ 100 metres par jour. C'est un instrument très délicat et d'un maniement difficile.

Nouvel étalon en invar. - Au cours des études que nous avons poursuivies, M. Benoît et moi, pendant plusieurs années sur les étalons géodésiques de divers pays, nous avons eu de nombreuses occasions de noter les avantages et les inconvénients respectifs de chacun d'eux, et nous avions esquissé depuis longtemps les plans d'un nouvel appareil, lorsque la découverte de l'acier-nickel à faible dilatation, l'invar, comme on est convenu de nommer cet alliage, vint donner à nos projets un sens plus précis. Nous les élaborâmes alors complètement, et nous pensàmes pouvoir mettre à la disposition des géodésiens un étalon unissant la précision à la légèreté et à la robustesse, conditions secondaires dans un laboratoire, mais qui deviennent primordiales en campagne. M. le général Bassot, à qui nous avions communiqué nos projets, nous confia, en 1898, la mission de faire construire, pour le Service géographique de l'Armée fran§aise, une règle géodésique de 4 mètres en alliage invar, dont je vais donner la description sommaire( 1 ).

L'étalon consiste en une barre à section en $\mathbf{H}$, de $\mathbf{4 0}$ millimètres au côté, aỵant son tracé dans le plan des fibres neutres. La règle est assez robuste pour qu'il suffise de la porter en deux points, toujours les mêmes. ce qui dispense d'avoir à effectuer le difficile alignement d'une sirie de supports, nécessaires dans le cas d'une règle flexible. Les deux points sur lesquels repose la règle consistent en un sup- 
port fixe et un rouleau, muntes a linterieur diune robuste d’aluminium qui enferme completement la règle, à la seule tion des petites fenètres destinées a viner les traits de l'etalon, et d. deux ouvertures laissant passer les pieds d'un niveau. que lon fait reposer -ur sa surface supérieure.

Deux thermomètres sont placés dan- le crenx d. la rente. Leturs réservoirs sont logés dans des blocs d’aluminium. qui, tant juintifs avec la règle, et enfermés avec elle dans la boite, prennent bien sa température.

Cette règle, complètement étudiée au Bureau international après son achèvement, a été emportée récemment dans la Ripulilique d. l'Équateur, où elle doit servir à la mesure d'une base par les soins des officiers de la mission française. Elle a paru si pratique aux géodésiens que quatre autres étalons semblables ont été executes depuis lors; les barres ont été ébauchées aux acièries d'Imphy. et achevées par la Société genevoise.

Je ne saurais insister ici sur les simplifications que cette règle apporte aux mesures géodésiques. Il suftira de dire que la faible dilatabilité du métal et les précautions prises pour diminuer les erreurs de température, tout en assurant une précision supérieure à celle. des anciens appareils, permettent de supprimer divers accessoires très encombrants du matériel ancien, tels que les baraques de protection; gràce à ces simplifications, on peut estimer à la moitié le gain du personnel auxiliaire, employi surtunt it transporter du matériel, et à la moitié aussi le gain de tempi dan-leopérations. Ainsi le coùt de la mesure d'une base a liaide de a.th.

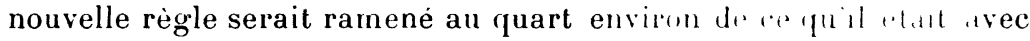
les anciens appareils. Mais nous avons $\mu u$, M. Benoit it moi, en perfectionnant d’autres méthodes déjà en usage, réaliser un z̧ain de temps incomparablement plus élevé (').

MESCRE PAR LES FILS TEXDES.

Irincipe de l/ methode. - A coté des méthodes utilisant des italıni ricrides en connexion avec des microscopes, methodes tr.. precises, mais compliquees et diune application contence, les pro-

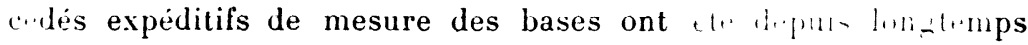
employés dans tous les cas ou une haute précision nétait pas J. de Phys., 4. série. t. V. Irril 1906.) 
tair. Mai los mitholes analogues ont été appliquées depuis longtemps sum in plus parfaites.

Lenlung- -uppriment les petites erreurs provenant des joints de la chaine: si on les appuie, à l'exemple du Service hỵdrographique de lat marim. $\mathrm{fr}_{\mathrm{r}}$ sur des table- bien dressées, et si, de plus, "nl lenr dont... -ion à peu prís constante, on évite d'autres causes d'incertitule. de telle sorte qu'on a pu, par des perfectionnement--110e-if, diminuer l'écart, primitivement énorme, de la préci-inn fommie par les deux groupes de méthodes.

La substitution de fils aux rubans, telle que l'avait proposée Goulier pour son télémètré. atténue l'effet du vent.

Tels étaient les faits dej. comnus, lorsque M. Jäderin eut, il y a

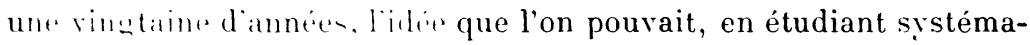
tiquement les meilleures conditions d'emploi des procédés rapides, leur donner une précision suffisante pour la topographie, et mème pour la géodésie, au moins provisoire, des pays neufs.

L'ítalon adopté par $\mathbf{~} \mathbf{l}$. Jäderin est un fil de $\mathbf{2 1}$ mètres de longueur et de $I^{\text {wm }}, \bar{\tau}$ de diamètre environ $\left({ }^{1}\right)$. Il porte, à ses extrémités, des réglettes divisées en millimètres sur une longueur d'un décimètre. Pour le transport, les fils sont enroulés; pour l'emploi, ils sont tendus sous un effort de 10 kilogrammes, obtenu au moyen de deux dynamomètres quactionnent dés aides placés aux deux bouts du fil. On remarquera que, si les fils sont soumis à une tension invariable, la distance rectilignede leurs points extrêmes est toujours la même.

Deux observateurs saisissent chacun l'une des réglettes, et l'amènent au contact d'un goujon vertical monté au sommet d'un trépied et portant une croisée de traits, qui remplace le fil d'araignée du micromètre dans les méthodes utilisant des microscopes. Lisant la position du trait de chaque goujon par rapport à la division de la réglette correspondante, les observateurs déterminent la distance des reprers mobiles. La base est jalonnée par une série de repères, alignnés et à peu prés équidistants, que l'on enlève à l'arrière, pour les reporter à l'avant de la section mesurée; l'équipe entière passe a in-i l'une portée à l'autre, jusqu'au deuxième terme de la base,

(1) La longueur frimitivement choisie etait 29 , m.tre- Mai- un l'abandonna bientot pour le multple le i mètres le plus wa-11. de maniere a permettre létalonnage du til à lialle d. regles géodé-ique- le inctres. 
dont on mesure la distance au dernier repère mubile au moyen dun ruban divisé.

Pour connaitre la température du fil employé à la mesure. Y. I.tderin déterminait chaque portée successivement au moven de deux fil. l’un d'arier. laautre de laiton, appliquant ainsi à sa méthode le principe de Borda et Lavoisier.

Les essais faits en divers pays à l'aidt des appareils imacrin - pror II. Jii derin montrèrent aux géodésiens que des méthodes d une grande rapidité pouvaient fournir des résultats d'une précision suffisante, dans bien des cas pour qu'on pût les appliquer partout, sauf pour les bases fondamentales. Il parut dès lors très intéressant de chercher à les perfectionner encore, et à les amener aussi près que possible de la précision des méthodes classiques employant des rèules et des microscopes.

Llyliration de linvar. - Lorsque les propriétés de l'invar furent suffisamment connues pour qu'on n'eût pas à craindre de sérieux déboires dans son emploi, nous songeâmes, M. Benoît et moi, à l'appliquer à la mesure des bases par les procédés rapides, et nous avions déjà fait quelques essais dans ce sens lorsque M. le général Bassot et $M$. Jäderin exprimèrent, indépendamment, le désir de poursuivre des recherches dans la même voie. Effectivement, des mesures faites en commun avec $\mathrm{M}$. le lieutenant-colonel Bourgeois et plusieurs officiers du Service géographique de lirmée française nous convainquirent bientôt qu'un gain réel de précision serait obtenu par l'emploi de l'invar, tandis que M. Jïderin érquipait, de son còté, l'expédition suédo-russe du Spitzberg avec des fils qui servirent, dès l'été 1899, à la mesure des bases principales de cette importante opération.

Les résultats furent de toutes parts si encourageants que nous pensàmes pouvoir aborder, avec de sérieuses chances de succès, en vue des opérations de la géodésie supérieure, l'étude des mesures par les fils, inscrites en 1900, par le Comité international des poids et mesures, au programme des travaux du Bureau, à la demande de l'Association géodésique internationale. Les résultats obtenus depuis lor ont dépassé notre attente.

Notre travail se divisa lui-mème en deux parties bien distinctes : d'une part, l'étude des fils; de l'autre, le perfectionnement des appareils servant à les utiliser.

L'étude des fils exigea des observations très nombreuses qui, à 
l'heure actuelle, se chiffrent par centaines de mille. M. L. Maudet et $\mathbf{M}$. A. Tarrade nous ont secondés avec beaucoup de dévouement dans ce labeur considérable. Pour les appareils eux-mêmes, la coopération de $\mathbf{M}$. Carpentier, qui a réalisé sous une forme élégante et en les perfectionnant encore les appareils dont nous avions établi les projets, nous a permis d'offrir aux géodésiens un matériel qui semble ne plus laisser beaucoup à désirer.

ÉTUDE DES FIIS.

Méthode. - Pour permettre de 'déterminer la longueur des fils à l'étude, il était tout d'abord nécessaire de constituer une base à laquelle ils seraient rapportés. Notre premier projet consistait à construire un véritable comparateur, de 24 mètres de longueur, dont l'élément principal eùt été une règle composée d'un nombre minimum de pièces solidement fixées bout à bout, et supportées sur un chariot solide, et susceptible de se déplacer sous deux microscopes. Mais, cette construction devant être très couteuse, il parutplus sage d'adopter, au moins provisoirement, une solution plus économique, bien qu'apparemment moins satisfaisante. La base que nous construisìmes dès le commencement de 1901 se composait d'une série de repères solidement fixés contre un mur épais, en sous-sol del'observatoire du Bureau international. Ces repères, parfaitement alignés dans tous les sens, et distants de mètres l'un de l'autre, jalonnent une base de 2\% mètres, avec un repère supplémentaire permettant de mesurer 20 mètres. Deux poulies montées sur billes, et situées extérieurement aux termes extrêmes, servent à supporter des cordes que l'on attelle au fil en étude, et auxquelles des poids de 10 kilogrammes assurent une tension constante. Les réglettes sont amenées simultanément contre les deux repères extrêmes, et les lectures, faites aux deux extrémités simultanément par deux observateurs, donnent la différence de longueur entre la base et le fil étudié.

La longueur de la base elle-même est déterminée par sections au moỵen d'une règle de mètres. dont le bord antérieur fait suite à la surface du repère fiy. 1. La règle porte, près de ses extremit八, d ux paires de traits, que l'on amène de part et d'autre du trait de repère correspondant; on lit, simultanément aux deux 
extrémités. à laide dun micromètre horizontal, la position des trois traits, ce qui fixe la distance des repères par rapport à la longueur de la règle.

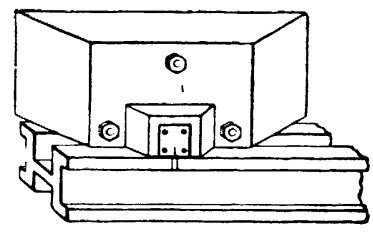

Fig. 1.

Nos premières mesures, faites au moyen de fils d'alliages divers, eurent seulement pour but de nous donner un premier aperçu de leur stabilité relative et des variations que leur faisaient subir les diverses manipulations auxquelles ils devaient être nécessairement soumis dans leur emploi. Puis, après des mesures poursuivies pendant près de deux ans, nous pûmes adopter un plan de travail bien défini.

Nous nous étions bientôt aperçus que la muraille portant nos repères éprouve, dans le cours du temps, des variations bien mesurables; mais, en même temps, nous avions reconnu que le plus gros de ces variations est une fonction bien définie de la température, c’est-à-dire que la muraille se dilate à très peu près comme un corps homogène. Son coefficient de dilatation, pour la détermi nation duquel nous avons déjà pu utiliser dans de bonnes conditions trois cycles annuels de la température, est de $7^{\mu}, 0$ par degré et par mètre, soit les trois cinquièmes environ de la dilatation du fer.

Quant aux fils eux-mêmes, nous avons été, dès le début de nos expériences, surpris de leur stabilité relative, c'est-à-dire de l'égalité approximative de leurs différences pendant une durée prolongée ; et, finalement, cette preuve étant faite en particulier pour les fils d'invar, nous avons concentré toute notre attention sur ces derniers.

Les expériences préliminaires étant terminées, et nous ayant montré ce qu'on pouvait attendre des fils comme précision et perman'm'e nous abordâmes un programme de recherches plus minutieuses. W'abord, nous modifiâmes la construction des réglettes terminales de manière à mettre l'extrémité des traits dans le prolongement de laxe du fil (fig. 2. On évite ainsi les différences de la longueur 
apparente du fil. c’est-à-dire de la distance de deux traits donnés. suivant l'inclinaison de la face divisée. Or la construction des repères de notre base, installés de manière à faciliter autant que possible les comparaisons avec la règle, nous oblige. dans la détermination des fils. à tourner vers le haut l'arète divisée des réglettes. tandis que cette arète est dirigée horizotalement ou plus ou moins obliquement vers le bas dans les mesures sur le terrain. Avec les réglettes des anciennes constructions, qui sont traversées par le fil, et dont le bord est excentrique, la différence de longueur est d'environ $0^{\mathrm{mm}}, 3$ dans les deux positions extrêmes des réglettes.

-

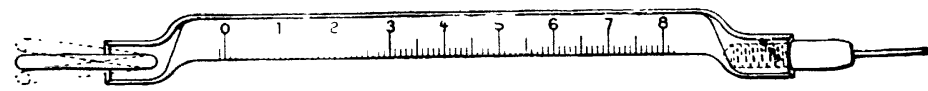

FIli. :.

Une série de douze fils d'invar, munis de réglettes du nouvean modèle, nous sert maintenant au contrôle permanent de la base, assuré de la manière suivante :

Dans les jours qui précèdent et dans ceux qui suivent la mesure absolue de la base à l'aide de la règle, chaque fil est comparé 200 fois à la base; les valeurs relatives de la base et des fils sont alors ramenées à une température de référence, ainsi que les résultats des déterminations absolues. La combinaison des deux nombres ainsi obtenus donne la valeur moyenne des fils de contrôle, que l'on suppose constante jusqu'à une prochaine détermination absolue, et qui sert, dans l'intervalle à donner. pour chaque jour d'observation, la valeur de la base. Après chaque mesure absolue, on fait la rectification nécessaire de la valeur admise pour les fils.

En tenant compte de la lente variation de l'invar avec le temps. variation qui, pour un alliage bien étuvé, arrive rapidement à n'ètre plus que de l'ordre du millionième par année, et dont la marche est bien connue, nous avons trouvé que la rectification it fair". d'une détermination a la suivante de notre base, est en moyenne sensiblement inférieure au millionième de la longueurmesurée. On peut donc être certain que le contrôle de la base par les fils est toujours largement assuré au millionième près, quantiti: encore très petite en géodésie.

Cela étant, il est intéressant de comparer la valeur de la base 
déduite de sa comparaison avec des fils avec sa valeur calculée en partant de sa température, déterminée à l'aide de six thermomètres enferme's dans des blocs de ciment faisant corps avec la muraille. Le résultat ainsi obtenu est remarquable; souvent, pendant trois ou quatre mois, les deux séries de valeurs ne diffèrent pas de plus de $\stackrel{-1}{\prime}$. 3 centièmes de millimètre, soit d un millionième des longueurs comparées. Puis, de temps en temps, et notamment aux chancrments de saison, il se produit un petit mouvement, d'un ordre un peu supérieur, mais qui est toujours resté jusqüici inférieur au dixieme de millimètre.

L.a comparaison des fils avec la base a lieu, au minimum, une fois par semaine, et souvent deux ou trois fois. Dans chaque comparaison, il est fait dix mesures indépendantes, avec échange des observateurs. Les lectures sont effectuées à l'aide d'une loupe moyennement grossissante.

I a connaissance de la longueur de la base permet, à son tour, de déterminer des fils de longueur inconnue, soit seulement en vue des mesures géodésiques auxquelles ils doivent servir, soit pour connaitre l'effet des traitements auxquels ils sont soumis dans un but d'intérêt général.

Les premières de ces déterminations ont une grande importance pour les opérateurs qui auront à employer les fils; mais il est sans intérit d'en parler ici.

Je vais, au contraire, résumerles résultats d'ordre général.

Résultats. - Un certain nombre de fils ont d'abord été soumis à des tensions croissantes, et mesurés dans les temps de repos. La déformation n'atteint sa valeur limite qu'au bout d'un certain temps, fonction de la charge. Insensible sous 20 kilogrammes, elle commence à se faire sentir sous 30 kilogrammes, puis elle augmente rapidement avec la charge, de manière à atteindre, sous 60 kilogrammes $\left(27 \mathrm{~kg}: \mathrm{mm}^{2}\right)$, un allongement de 1100000 en vingtquatre heures. Cette dernière valeur de la variation est celle des fils l...l .rouis. Un fil moyennement dur éprouve des changements un peu plus forts.

Des enroulages répétés du fil, ramené à la forme dune couronne dun diamitre de ว̈) centimètres, font disparaitre en partie les allongements produits par des tensions excessives. Mais la limite est tries vite atteinte, et, après une dizaine d'enroulages et de déroulages au maximum, le fil prend sa valeur définitive. On peut alors 
lui faire subir de tres nombreux enroulages sans voir sa longueur se moditier.

L'absence d'effets appréciables pour des enroulages répétés un grand nombre de fois consécutivement ne résout pas la question de la conservation de longueur du fil, enroulé ou étendu. Nous avons consacré un très grand nombre d'observations à cette dernière étude. à laquelle un ecinquantaine de fils ont participé et dont les résultats, sont maintenant appuyés sur vingt mille lectures environ. Après avoir déterminé. aussi bien que possible, la longueur d'une série de dix fils, nous les avons enroulés et repris au bout d'un mois. La même opération a été répétée sur dix autres fils, restés enroulés pendant six semaines, et ainsi de suite. Les repos les plus longs que nous ayons fait subir aux fils enroulés ont été jusqu ici de huit mois. Nous n'avons trouvé que très exceptionnellement des variations supérieures au millionième par rapport aux fils restés constamment étendus pour le contrôle de notre base.

Ces dernièré constatations sont de la plus grande importance pour lavenir des méthodes rapides de mesure des bases. Elles sont décisives, en effet, au sujet de la possibilité d'emporter en campagne des fils dont la longueur a été déterminée dans un observatoire, sans quion ait à mesurer de nouveau leur équation sur le terrain, comme on l'avait toujours jugé nécessaire tant qu'on n'avait pas la preuve expérimentale de la conservation de cette équation.

Assurément, une vérification sur une courte base, voisine du terrain de la mesure géodésique proprement dite, fournira un contrôle précieux, qu'on voudra s'assurer tant qu'on le pourra sans une trop grosse dépense; c'est là surtout que la règle rigide en invar trouvera son application dans l'avenir. Mais, dans tous les cas où une telle vérification sera coûteuse, on préférera contrôler la valeur du fil servant à la mesure par sa comparaison avec deux ou trois autres fils, également déterminés dans un observatoire. Cette comparaison pourra être faite simplement en prenant, comme points fixes passagers, deux repères mobiles, tels que ceux qui seront décrits dans un instant, ou, de préférence, deux repères fixés contre une muraille ou sur des piliers.

Les résultat - fui viennent d'être rapportés ont été obtenus seulement pour de-tils traités avec de minutienses précautions, soustraits aux secoussi's, et inroulés toujours -ur un diamètre minimum de so centimètres ramenant le fil à trís peu près dans ses plis 
naturels. L'enroulage sur des diamètres sensiblement plus petits conduit à dépasser la limite élastique, et entraine des changements bien mesurables.

Les secousses violentes modifient aussi la longueur des fils, même s il n'en est résulté aucune courbure durable. Les changements consistent toujours en une contraction des fils soumis à de fortes secousses lorsqu'ils sont étendus, et généralement en un allongement des fils frappés tandis qu'ils sont enroulés. Ces modifications sont dues certainement à des glissements, qui rapprochent le fil de son état d'équilibre mécanique.

L'effet des secousses s'atténue rapidement à mesure que leur nombre augmente; et, pour mettre les fils complètement à l'abri des changements une fois leur étude commencée, nous les battons vigoureusement sur le plancher, avant même de les remettre au constructeur pour $y$ fixer les réglettes terminales. Puis, celles-ci étant adaptées aux fils, nous les soumettons à une traction de 60 kilogrammes, d'une durée de vingt-quatre heures, destincée à nous assurer de la solidité des attaches et à annuler les effets possibles des tractions normales sur des fils dont les réglettes n'auraient pas pris leur assise; nous les battons encore 200 fois, après quoi ils sont prèts à être mis à l'étude.

Après chacune des manipulations qui viennent d'être indiquées, les fils sont mesurés de manière à nous permettre de découvrir des changements anormaux, qui nous conduiraient à rejeter un fil présentant un défaut de construction. Je m'empresse de dire que de telles anomalies sont extrêmement rares ; la presque totalité des fils éprouvent, dans la série des traitements que nous leur faisons subir, des variations qui s'écartent très peu d'une valeur moyenne, dont une longue statistique nous a donné la valeur.

Pricision des mesures par les fils. - Il me reste un mot à dire sur la précision que permettent les mesures par les fils, telles que les nombreuses comparaisons effectuées sur notre base nous l'ont fiit connaitre.

Les lectures sont inscrites au dixième, ou au plus au vingtième de millimitre. Sur un groupe de dix lectures, faites successivement dans diverses régions des réglettes, les écarts extrêmes de $0^{\mathrm{mm}}, \mathbf{3}$ sont rares, et les moyennes successives de groupes indépendants cuncordent toujours à quelques centièmes de millimètre près. I ierreur moyenne diun groupe de dix comparaisons est de lordre 
du millionième. Chaque portée peut donc être considérée comme mesurée avec une précision suffisante en moyenne pour les besoins de la géodésie.

Toutefois certaines portées pourront, par le fait des erreurs de lecture. ètre erronées d'une quantité voisine du dixième de millimètre. Mais, si mème on supposait que l'erreur moyenne fùt de cet ordre de grandeur. la précision olıtenue serait encore bien plus que suffisante pour la mesure des bases. En effet, les erreurs de lectures, - thant fortuites, sadditionnent suivant la loi de la racine carrée : ainsi. après 100 portées, l'erreur ne devra être que le décuple de l'erreur d'une portée; donc, mème en supposant aux erreurs des lectures la valeur movenne de $0^{\mathrm{mm}}, 1$, trís supérieure à celle qu'elles possèdent en réalité, une base de 2.400 mètres devrait être connue avec une précision moyenne de 1 millimètre, précision très élevée si on la compare à celle du rattachement aux sommets des triangles adjacents ì la base. Le rapport des erreurs diminue à mesure que la base s'allonge, et il ne faut pas oublier qu'une base de 2.100 mètres serait très courte.

On voit donc que, malgré leur rusticité, les lectures faites sur les fils sont amplement suffisantes pour les besoins de la géodésie la plus précise. Mais ce résultat n’est obtenu que si les erreurs des lectures sont réellement fortuites et ne comportent aucun élément systématique appréciable. Or cliaque observateur possède une équation personnelle, qui le conduit à attribuer à un trait rapporté à une division une position erronée en movenne dans un sens díterminé. Mais cette erreur systématique est complètement éliminée si l'on échange les observateurs dans chaque mesure, que l'on fait ainsi en doul,le, ou simplement si on les échange périodiquement, après un nombre déterminé de portées, dix par exemple. Dans le premier cas, l'erreur est iliminée dans chaque portée; dans l'autre, elle ne lest que dans chaque période complete. Pour la détermination d'un til ou pour le contrôle d'une base de 21 mètres comme celle du Bureau international, l'échange des observateurs est nécessaire: tandis que, dans la mesure d'une base sur le terrain, l'échange piriodique, qui constitue une grosse économie de temps, est parfaitement suffisant, puisque la connaissance individuelle des portées est indifférente, leur somme ayant seule de l'intérèt.

Les indications qui précèdent montrent a l'évidence qu il serait tout à fait superllu de chercher à perfectionner encore beaucoup le 
mode de lecture, comme quelques observateurs l'ont proposé, par exemple en adaptant des verniers aux repères, ou en lisant à l'aide de microscopes. Tout ce qui, dans la mesure d'une base, cause une perte de temps, peut entrainer des erreurs, et rend la mesure plus couteuse; il n y a donc lieu de chercher que les perfectionnements réellement utilis. et nonà augmenter encore la précision de tel ou tel élément de l' ":" ration, a partir du moment où cette précision est plus que suffisante.

Dans ce qui précède, il a été possible d'envisager les erreurs des lectures en elles-mêmes, sans tenir compte des variations de longueur du fil dues au changement de la température et an défaut de connaissance de cette dernière. S’il s’agissait des métaux ou alliages usuels, une discussion ainsi limitée n’aurait eu aucun sens. En effet, la dilatabilité de l'acier étant un peu supérieure à un cent-millième par degré, il faudrait, pour connaitre la longueur d'un fil d'acier au millionième près, déterminer sa température moyenne au dixième de degré près, ce qui est tout à fait impossible en campagne. Mais, avec l'invar d'une coulée bien réussie, la dilatation est absolument négligeable. Les aciéries d'Imphy peuvent en effet fournir assez régulièrement des fils dont la dilatation est ile l'ordre de $0^{\mu, 1}$ à $0^{\mu,}, 2$ par degré et par mètre, dilatation ö0 à 100 fois plus faible que celle de l'acier. Dans ces conditions, il faudrait commettre, sur la lempr..rature du fil, une erreur de $\breve{\jmath}$ à $10^{\circ}$ pour (jü le chiffre du millionieme fùt affecté dans le calcul de sa longueur. Une aussi grande latitude laissée à la mesure de la température lui enlève toute espiece de difficulté.

Les fils d'invar sont soumis, comme il a èté dit, aux causes naturelles de variation de eet alliage, qui consistent en une tendance constante vers un équilibre chimique de plus en plus parfait, et se manifestent par un allongement progressif avec le temps. Mais, à partir du moment, bientót atteint, oi la variation arrive a itre de lordre d'un millionième par anten. Il tra ntrimement facile. par des comparaisons faites avant et apen une rampange de mesures, de déterminer, pour chaque époque, la valeur des til arice une précision plus que suffisante. Je renverrai, pour des induatim-plun pletes sur les variations des aciers au nickel, at des publications anterieures $(1)$.

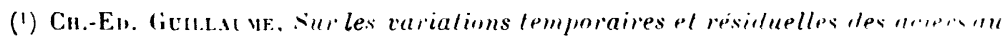

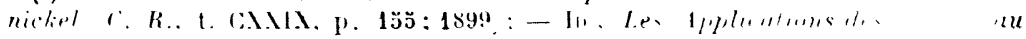

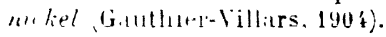


Nous avons eu en vue, dans la constitution du matériel de campagne, à la fois la rapidité de la manœuvre et la précision du résultat. Lemploi des fils dinvar ayant augmenté dans une très forte proportion la sécurité des mesures, il devenait nécessaire de perfectionner, dune manière correspondante, le reste du matériel combiné par M. Jäderin. Nous croyons y avoir réussi sans rendre la manœuvre plus difficile et sans arriver à des complications lui enlevant sa rusticité. Sur bien des points, le nouveau matériel est même plus simple que le matériel primitif de $\mathbf{M}$. Jäderin, et permet de pousser l'économie du temps et du personnel au delà du degré où il l'avait conduite.

Tenseurs. - Il est tout d'abord nécessaire d'assurer aux fils une tension constante, égale à celle sous laquelle leur équation a été établie. Si, au voisinage de la tension normale de 10 kilogrammes, la charge varie de 1 kilogramme, la longueur apparente du fil ( $\left.{ }^{1}\right)$, c'està-dire la distance rectiligne de deux traits homologues des réglettes, se modifie à très peu près de 1 millimètre. Les erreurs systématiques de la tension doivent donc être éliminées à quelques grammes près, et les erreurs fortuites à quelques décagrammes.

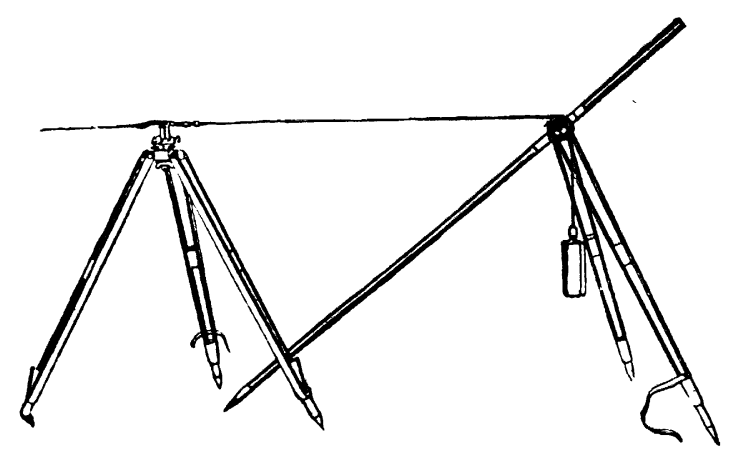

FI:. 3.

Dans la plupart des mesures faites jusqu ici, lit tension des fils ou des rubans était obtenue par des dynamometres. Iaais une étude rapide de ce mode d'opérer nous a bientôt montré qu'il était très

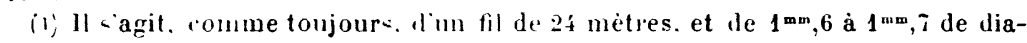
mètre. 
peu -uir. Nous avons donc adopte le mode de tension par des poids attelés à des cordes souples passant sur des poulies à billes. Ces poulies fiy. 3 sont portées par des chapes montées sur des piquets inclinés. quelion soutient par deux jambes de force articulées. On remarquera que, au voisinage d'une inclinaison de $\mathrm{s} 0$ grades. la résultante des efforts exercés sur la poulie par les deux brins de la corde est dirigée suivant la longueur du piquet, de telle sorte que les jambes de force n'ont, pour ainsi dire, aucun effort à supporter. Si les conditions du terrain l'exigent, on peut mème les démonter; chaque piquet est alors maintenu en place par un auxiliaire, duquel on n'exige que de l'immobilité pendant la durée des observateurs.

Reprires moliles. - Nous nous sommes proposé de donner aux reperes mobiles une forme telle qu'iis puissent ètre amenés rapidement et trés exactement dans la position que chacun d'eux doit occuper dans la ligne de la base; nous les avons disposés aussi de manière à leur faire porter les accessoires destinés à la mesure des

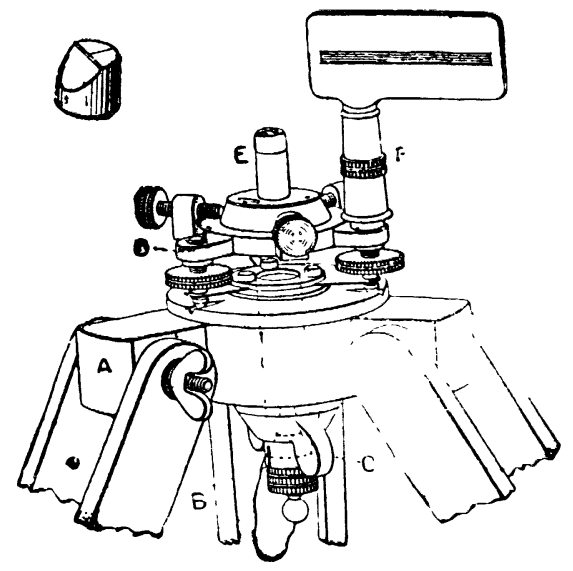

Fig. 4.

pentes, sans que l'on ait besoin d'avoir recours, comme on l'avait fait juscyu ici à des installations auxiliaires. Enfin, nous avons admis la possibiliti de déterminer, par une opération simple. un point du sol situe verticalement au-dessous de la croisée de traits qui constitue le print d. l'espare auquel viennent se joindre les dew cessives. 
Voici comment ce programme a té mis à exécution : L'n solide trépied de bois - i. i porte, à sa partie supérieure. un plateau $A$ percé d'une large vuverture verticale. Ln tube cyllindrique $B$, fixé au-densous d'une plaque-crapandine. et portant à sa partie inférieure un écrou à oreilles C, est serré contre le plateau de bois, par rapport auquel il peut. d'ailleurs. occuper des positions quelconques dans un espace de quelques centimètres.

La plaque-crapaudine est surmontée d'une tallette de bronze ID, montée sur trois vis calantes maintenues en place par des ressorts, et qui est munie. à sa périphérie. de trois vis radiales permettant un déplacement micrométrique d'une piece $\mathrm{F}$. composée d'une embase portant un niveau et surmontée d'un goujon vertical. Ce goujon est terminé par une pastille en un alliage blanc, dur et inoxydable, au centre de laquelle se croisent les traits rectangulaires du repère, ou par une plaque semi-circulaire (détail) portant un seul trait radial.

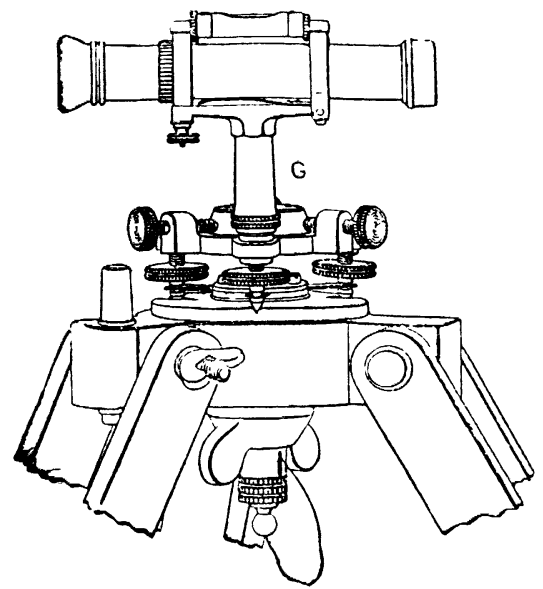

FIG. 5.

Le goujon est percé d'un canal central, dans lequel descend la cordelette de suspension d'un fil à plomb permettant de marquer un point du sol situé verticalement au-dessous de la croisée de traits, et qui. lorsqu'il est sans emploi, reste vissé de bas en haut, dans le tube de la plaque-crapaudine.

La tablette supérieure est munie, en outre, d'un goujon latéral 
fixe. qui peut supporter alternativement une mire $F$ et une lunette de nivellement fig. ¿ servant, déterminer la par la visée de la mire, à diffrince de hauteur de deux trépieds consécutifs.

Pur determiner l'alignement des repères. on pose. sur le goujon mubile du dernier repère placé, une petite lunette qui sert à viner à la h,i- une mire lointaine, dans la direction du deuxième trine le la bar. et le goujon du repère que lon met en station. Les imawes de ces deux objets se superposent quand le repère est définitivement en pl..

- Hemroulage cles fils. - Nous avons établi pour l'enroulage des fils, en vue de leur transport, un tambour cons-intant /'./.6) en une poulie légère, avec moỵeu et jante d’aluminium réunis par des rạons en tubes d'acier: la jante est munie, à sa partie périphérique, de deux séries de crochets auxquels viennent se fixer les extrémités des fils; la longueur des crochets est telle que les fils arrivint tangentiellement au tambour.

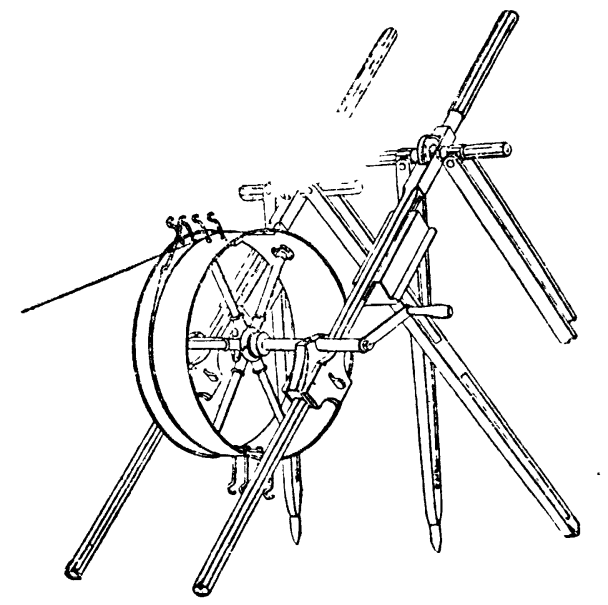

FIG. 6.

L'axe de la poulie est amovible; il repose, pour l'enroulage et le déroulage, sur deux paliers de bois, également amovibles, qu'on s erre, à l'aide d'une clavette, sur deux piquets tenseurs placés l'un à côté de l'autre, et qu'on enlève ensuite.

Pour le transport, le tambour est enfermé dans une caisse uu il est retenu, par ses bras, sur trois cales de bois. Liaxe et les paliers trouvent également place dans la caisse. 
II. Carpentier a modifié ce premier instrument en montant l'arbre a demeure dans les coités dune caint fiø. 7 . qui lui servent de paliers. De cette façon. l'enroulage ou le déroulage s'effectuent sans que l'on ait a faire intervenir les piquets tenseurs comme supports.

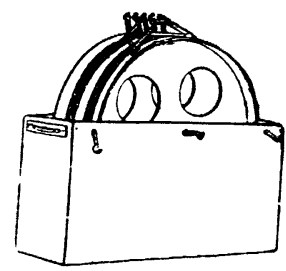

Fig. 7.

Accessoiresdivers. - Le matériel de mesure dune base comprend enfin :

$1^{\circ}$ Des repères mi-fixes que l'on enfonce dans le sol à tout arrêt prolongé des mesures ;

2. Un gabarit en câble toronné, muni de deux bagues marquant un intervalle de $\mathbf{2 4}$ mètres et servant à placer les repères mobiles à la distance voulue. Il est accompagné de deux piquets de bois que tiennent les opérateurs;

$3^{\circ}$ Les étalons nécessaires pour permettre de mesurer l'appoint. c'est-à-dire la distance, inférieure à 24 mètres, qui sépare la position du dernier repère mobile du deuxième terme de la base. On peut employer, dans ce but, soit un ruban de 12 mètres, soit un fil de 8 mètres auquel succède un ruban de 4 mètres. Le ruban doit posséder les traits marquant tous les décimètres, et des divisions millimétriques du premier et du dernier décimètre;

$\mathbf{1}^{\circ}$ Une série de mires, que l’on place au théodolite dans la ligne de la base. et qui servent ensuite à aligner les repères mobiles ;

$\dddot{\partial}^{\circ}$ Des loupes, des thermomìtres et des outils divers.

Emploi res appareils. - Pour la manveuvre sur le terrain, le personnel se divise en deux "yuipes : la première pose les repères, la seconde mesure leur distance deux à deux.

La première équipe se compose de trois opérateurs, dont l'un remplit les fonctions de chef. Celui-ci reste à l'arrière de l’équipe, et tient une des extrémités du gabarit dont l'autre extrémité est portée par un auxiliaire. I.e d.u se réglant. pour la distance. sur une marque du gabarit, et pour laali- 
gnement. sur les indications du chef. Lin certain nombre de porteurs. attachés à l'équipe, ramènent à l'avant les trépieús devenus inutiles à l'arrière de la section mesurée de la base.

Lorsque tout fonctionne normalement, un repire molbile doit ètre complètement réglé en une minute et demie environ.

La deuxième équipe comprend un secrétaire chef d'équipe. deux olservateurs et deux porteurs. Le secrétaire dirige lopretion: il inscrit les lectures que lui indiquent les observateur. . . . d.tn- la règle, détermine la pente.

Dans des conditions moyennes de terrain, le travail des deux équipes est à peu près synchrone, de telle sorte que chaque équipe est constamment occupée. Cependant, comme l’une ou l'autre peut, en raison de difficultés particulières, prendre un retard momentané, il est nécessaire, si l'on veut éviter les à-coups, de laisser entre elles au minimum deux ou trois portées libres.

Cette condition fixe le nombre de repères mobiles qu'il faut avoir à sa disposition, si l'on veut pouvoir travailler commodément. En comptant les trépieds de la portée que l'on mesure, le repère laissé à l'arrière comme contrôle, celui que l'on met en place et ceux qui sont sur le chemin de l'arrière à l'avant, on voit que, pour opérer commodément, il est utile de posséder de 8 à 10 repères.

Quant au personnel, il se compose de dix à duuze hommes. dont quatre, savoir le chef de la première équipe, le secrulitr et les deux observateurs, doivent posséder une instruction professionnelle. On n'exige des autres qu'une suffisante habileté manuelle, de l'endurance et une attention soutenue.

Conclusions.

Nous avons vu que la mesure des bases par les fils. gràce aux progrès réalisés dans ces dernières années, suffit aux exignences de la géodésie supérieure. D’autre part, la vitesse de marchre th incom-

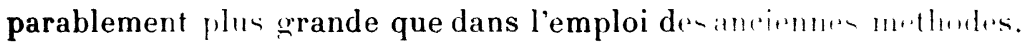

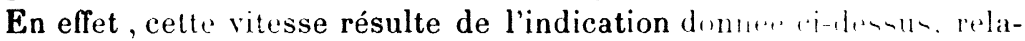
tivement à la mesure d'une portée. Si l'on admet quill lum travailler san- interruption, à raison de denx porfies in troi-minutes. la rapidit. de la mesure sera de lordre dı hilumielre à liheure. Ce .hufr.. est un peu exagér. parce qu il faut cumpl.r aver des retards inevitables dans le travilil le l'une ou l'autre équipe. surtout lorsque

J. de Phys., f́ série. t. V. Arril 1906., 
le personnel est fatigué par un labeur soutenu. Mais un personnel de dix à douze hommes exercés doit pouvoir atteindre, en bon terrain et par le beau temps. une vitesse de $\ddot{\partial}$ à 6 kilomètres par jour, en $y$ comprenant le repérage aux extrémités de la base et en un ou deux points intermédiaires (').

Nous avons vu que les anciennes mesures par la règle bimétallique exigeaient une cinquantaine d'hommes, et donnaient une vitesse de $\mathbf{1 0 0}$ portees par jour, solt $\mathbf{4 0 0}$ mètres. Les nouveaux procédés réalisent donc, par rapport à ceux qui utilisent la règle bimétallique, une économie supérieure à $980 / 0$.

Cette transformation dans les méthodes de mesure des bases doit nécessairement réagir sur l'ensemble des travaux géodésiques. Tandis qu'autrefois on diminuait autant que possible le nombre des bases à mesurer, on n'hésitera pas, dans l'avenir, à mesurer une base toutes les fois que cette opération présentera une utilité quelconque; en général, on devra s'imposer de ne plus dépasser un nombre restreint de triangles subordonnès les uns aux autres sans venir chercher un repérage sur une base.

La facilité d'un tel contrôle simplifiera, à son tour, la mesure des triangles. Comme les erreurs de ceux-ci s'échafauderont beaucoup moins qu'autrefois, on pourra être moins exigeant à leur égard, sans craindre de compromettre l'ensemble d'un réseau. Ainsi, la transformation opérée dans la mesure des bases modifiera profondément tout le travail des géodésiens.

Les avantages de l'emploi des fils d'invar, que nous avons indiqués, M. Benoît et moi, dans une série de publications provisoires faites à l'occasion des sessions du Comité international des poids et mesures et de l'Association géodésique internationale $\left(^{2}\right)$, ont attiré l'attention des services intéressés, et ont valu, au Bureau international, de nombreuses demandes d'études. Un grand nombre de bases géodésiques ont été mesurées, dans ces dernières années, ou

Diun une mesure à laquelle j'ai èté appelé à coopèrer, la Commission Gu...le-nulu suisse a déterminé récemment la distance, supérieure à 20 bilo-

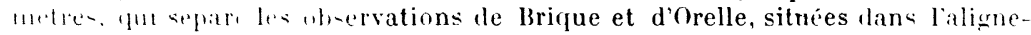
u1 nt du tunnel du - -muphn. Malugré les difficultés résultant du traval à l.t

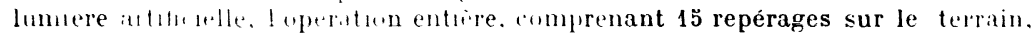

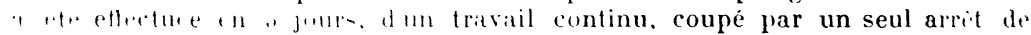

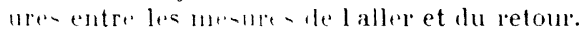

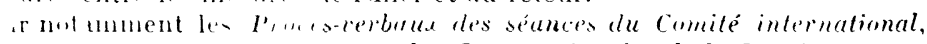

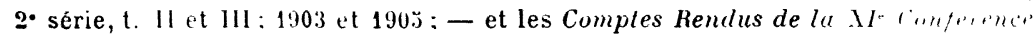
géodésique intrruntionale, réunie à Copenhague en aoùt 1903. 
JAFFE. - COXDLCTIBIITE DE LÉTHER IL PÉTROI.E Q.: sont en cours de détermination, à l'aide des fils dont nous avons déterminé l'équation et du matériel dont on vient de lire la description. Cet ensemble de travaux, qui se poursuivent actuellement dans les pays les plus divers. achèvera de faire connaitre les avantages que lion doit réellement attendre des nouvelles méthodes. 